\title{
Research on the Relationship between Values and Mental Health of College Students
}

\author{
Yanrong Zhai \\ Chengdu University of Information Technology, Chengdu, Sichuan, 610100
}

Keywords: college students; value; mental health

\begin{abstract}
Values are the basic views of individuals on people and things in the world around them. They are a series of the most basic criteria on which people make judgments that are or are not, meaningful or meaningless, worthy of acceptance, or unworthy to accept. The correct values can promote the improvement of college students' mental health, and the healthy psychology is the basis for the formation of correct values for college students. The purpose of this paper is to suggest that a variety of effective methods should be taken in the education of college students' mental health to guide them to establish correct values.
\end{abstract}

\section{Introduction}

Values are people's concepts about the importance of things, and they are the criteria for judging and selecting objects based on their importance to the subject. Values are often regarded as a core concept in the individual personality system and spiritual system. They play an important role in describing, explaining, predicting, and guiding individual behaviors. At the same time, values are also important measurement indicators for social development and cultural construction. In addition, values are also a concern of various humanities and social sciences. Schwartz defines basic values as trans-objective goals, which are of different importance and are guiding principles for the life of a person or a group. He also believes that basic values are organized into a coherent system that can be used to construct and help explain individual decisions and attitudes. Blankenship et al. also found that values are rarely attacked and defended, so people can indirectly change their attitudes by changing their values. Values are closely related to people's beliefs and pursuits and behaviors. In the study of psychology and other disciplines, there is a general consensus that values have a guiding role in overall behavior. However, there have also been studies on whether personality and values can predict the behavioral outcomes of people. It has been found that doctors' work values, whether used alone or in combination with personality traits, do not clearly predict professional choices.

Values are very complex psychological phenomena. They are a multi-dimensional and multi-level conceptual system. They are also the psychological tendency systems that people use to distinguish between good and bad standards and guide behaviors. They provide sufficient reasons for people to think that they are legitimate behaviors. They are impregnated with the entire personality dominates human behavior, attitudes, opinions, beliefs, and ideal inner dimensions. The author regards life values, life values, moral values, political values, learning values, and professional values as the six aspects of student values, and investigates the relevance of different levels of mental health of college students to their values.

\section{The Psychological Connotation of Values}

Kluckhohn (1951) considers values to be explicit or implicit about what is worthwhile. It is a feature that distinguishes differences between individuals and groups. It affects people's ways of behavior, means, and purposes. It is an individual's basic view of people, things, and things in the world around them. It is a series of the most basic criteria or criteria based on which people make judgments that are right or wrong, meaningful or meaningless, worthy of acceptance, or unworthy to accept. 
From the perspective of content, values include value goals, value measures, and value evaluation. Value goals are individuals who think, determine, and pursue goals that are important to their actions. They are questions about what people's actions are for. The value approach is the approach and methods that individuals take to reach value goals, and questions about how people act. Value evaluation is an individual's judgment on the value and value of objective things according to certain value standards. It is about the meaning and significance of human actions. Value evaluation is an important aspect of values. In social life, people evaluate the value of life and social behavior according to a certain value standard, and thus generate a sense of value and significance that is worthless, happy, and unhappy. The direction and extent of value goals and means and the corresponding social behaviors influence or promote or sustain or organize or change [1].

From a psychological structure point of view, values include cognitive factors, emotional factors and volitional factors. Cognitive factors refer to the perception and understanding of the value attributes of things, as well as the understanding and evaluation of the value of things. A person's understanding of the value of things at the same time, it also produced a corresponding emotional experience for him. When a person realizes that something can satisfy his or her needs, it will produce a positive emotion, or respect him, or sympathize with him or like him. The will factor refers to the psychological process of a person starting from his own value understanding, taking measures based on his selective emotional experience, taking measures, overcoming difficulties, and pursuing what he considers valuable or realizing his own value goals. The factors that influence the formation of values include individual needs, self-awareness, and life experience. Differences in values between people are caused by differences in their physical, psychological and living environments, and life experiences. These values in turn affect people's behavior, emotions, and personality development.

\section{Relationship between College Students' Values and Mental Health}

Many college students' psychological problems are caused by individual value conflicts. At present, our undergraduates are in the process of conflict, blending, and blending between traditional culture and modern culture. They are often in the midst of the choice of value, which is the emergence of value conflicts. It includes ambiguous value orientation, bias in value evaluation, unbalanced value identification, and misplaced values [2]. This kind of value conflict is manifested as the individual's psychological conflict. The conflict cannot be resolved in time. The individual will feel insecure, cause emotional problems, and develop into psychological problems. For example, in the psychological consultation and counseling of college students, the root causes of inferiority, arrogance, embarrassment, emptiness, dual personality, suicide, self-closedness, blind conformity, rebelliousness, selfishness, and enjoyment are the essence of such psychological problems. Inferiority and self-esteem are self-evaluations that are not correct, excessive self-denial or excessive self-affirmation, and a kind of arrogant or self-defeating emotional experience. The reason for this is that it only focuses on temporary surface interests, and it prides itself on superior status and isolation. The emptiness is caused by undue emphasis on personal gains and losses, and the misconception that personal requirements are not satisfied and all things are grayed out. The formation of dual personality is a comparison of two mutually antagonistic value evaluation standards for the undergraduate discipline and self-discipline. The reaction to growth and decline; and suicide is to a certain extent caused by the irrationality of factors such as the objectivity and multidimensionality of value appraisal. This irrationality is caused by individual's wrong attitude and cognition to the current frustration.

The objective factors that affect the mental health of college students are through the individual's values. There are many factors that affect the psychological health of college students, including biological factors, family environment and parenting methods, school conditions and interpersonal relationships, as well as social regional culture and other objective factors, as well as subjective factors such as temperament, personality, and emotion; The environment, in the face of the same pressure and frustration, different college students have different psychological feelings. In fact, it is 
different in their value orientation. The discussion of psychological health by scholars of all ages, both at home and abroad, has mainly focused on four aspects: psychological state, growth and development, social adaptation, and ethics. It can be seen that values have an impact on mental health. First, maintaining a good mental state requires that you Self-knowledge, self-control, self-discipline, so that they maintain a happy mood, full of self-esteem, self-confidence. Second, having good social adaptability requires individuals to understand the outside world, actively govern the environment, properly handle interpersonal relationships, and some beliefs about adaptation such as tolerance, enthusiasm, friendship, and gregariousness are themselves values. Third, it has a positive growth and development trend, and requires individuals to grow and mature continuously, to self-improvement, and to self-improvement. This is a series of value concepts about self-determination. Fourth, with a noble ethical and moral spirit, Maslow suggested that people should work harder and better, that is, toward what most people call a beautiful value, toward serenity, kindness, heroism, integrity, love, selflessness, Good progress. The ethical standard of mental health is to require individuals to have progressive values [3].

Coordinated, stable and correct values are a major condition for good mental health. Maslow, a humanistic psychologist, thinks that one criterion of mental health is whether there is a correct and reasonable philosophical view. Alice, the founder of cognitive therapy, also said that if a person has a reasonable philosophical view, then he is not easily plagued by various emotions. We in China also have stories that make things worse. The philosophical view and mood mentioned here actually refer to a kind of cognition and a kind of view on reality, that is, values. A mentally healthy person must have a harmonious and unified personality system. Values are the premise and foundation of personality formation. Values are part of the world outlook and make the core of the outlook on life. It best embodies people's purpose in life and their meaning in life. Because people's activity choices always have a process of thinking, and this kind of thinking process is always related to such five questions: need or not, like it or not, whether it is worth or not, whether it can be done or not, and whether it is implemented or not. These five issues are necessarily related to a person's cognitive value orientation. For example, when people think about issues, the consideration of whether things are worthwhile is determined by values. Values are a kind of cognition. They are the intermediary of stimuli and reaction. The input of external things is always through the conversion of cognition (mainly values), and the stimuli have different properties and different meanings. All kinds of cognitions are coordinated with each other and whether they are in a relatively stable state of mind. This determines the psychological balance of a person. It is the main condition for the normal operation of a person's mind and the background of a person's psychological process. If people's views on various issues are in a state of coordination, human psychology is in a state of balance. Conversely, when values are in a state of contradiction and instability, they are in an unbalanced state, which leads to cognitive dissonance, unpleasantness, and various psychological problems. For this reason, having a person with coordinated, stable, and correct values becomes a major condition for our good mental health [4].

\section{Suggestions}

Strengthen the mental health of college students and guide students to establish correct values. College students are often in the contradiction and perplexity between ideals and reality. Emotional excitement is more than rational thinking. Students lamented the contrast between school education and social reality. They also felt the contradiction between external education and their own inner value judgment level and moral level. Coupled with the influence of various erroneous values in society, they have shown swing and repetition in the formation of values. Therefore, in terms of loneliness, contradictions and perplexities in college students' study, life, interpersonal relations, employment, love, etc., we must correctly guide them to establish correct values of life, values of knowledge, professional values, interpersonal values, values of marriage, health values, etc. Values, as their behavioral concept system, guide their direction of action and have extremely important substantive significance and role in their personality development, social adaptation and mental health. 
When conducting values education, various effective guidance methods are adopted. The guidance of the values of college students is a complicated and arduous task. It is often difficult to work with a single fixed approach. Practice has shown that for a college student's physical and psychological development, taking various forms of education can receive good results. From the perspective of the subject, multi-level education should be adopted. Among the values of college students, there are gender differences, grade differences, arts and science differences, individual differences, etc. Different methods should be implemented according to different targets and different requirements should be put forward. In terms of content, we should focus on the theme of values, make appropriate changes according to different needs, and conduct three-dimensional education [5]. Through the in-depth development of various special education programs, they are encouraged to establish correct values. In form, it should be as diverse as possible. If you always use a form, they will get bored. Because values education itself is rich in content, while college students have a strong sense of curiosity, like to revive. Therefore, adopting a lively and active form of education, a form that students like to hear, and integrating science, knowledge, and interest together will enhance the persuasiveness, appeal, and appeal of education.

\section{Conclusion}

University is an important place for cultivating high-level talents. College students are also at an important stage in the formation and improvement of values. Therefore, we should use every opportunity to educate university students so that they can form positive and correct values (such as paying attention to character, human relations, family, public welfare, etc.). Correct and positive values will allow us to adopt a positive coping style in the face of pressure and frustration, and less to adopt a negative coping style. So as to eliminate or reduce the factors that affects the mental health of college students, so that college students can grow healthily and happily.

\section{References}

[1] Jin Shenghua, Zheng Jianjun, Xin Zhiyong. The Structure and Characteristics of Contemporary Chinese Values[J]. Chinese Journal of Psychology, 2009, 41(10):1000-1014.

[2] Xin Zhiyong, Jin Shenghua. College Students' Value Concept and Value Structure[J]. Studies in Higher Education, 2006, 27(2): 85-92.

[3] Schwartz, S. H. Boehnke K. Refining the Theory of Basic Individual Values[J]. Journal of Per-sonality and Social Psychology, 2012, 103(4):663-688

[4] Blankenship, K. L. \& Wegener, D. TCircumventing Resistance: Using Values to Indirectly Change Attitudes[J]. Journal of Personality and Social Psychology. 2012, 103(4): 606-621.

[5] Taber, B. J. et al. Personality and values as predictors of medical specialty choice[J]. Journal of Vocational Behavior .2011,78(2):202-209. 